\title{
Teaching the role of mutation in evolution by means of a board game
}

\author{
André Eterovic and Charles Morphy D Santos ${ }^{*}$
}

\begin{abstract}
Background: The board game ACAGATATA simulates how randomness originates biodiversity. An individual (genotype ACAGATATA) produces offspring with a chance of error during DNA replication (mutation) at each generation, showing that random changes in ancestral genotypes may reflect on descendant phenotypes.

Methods: The game has three steps: (1) construction of a dichotomous diagram by submission of the parental DNA to successive copies in which the chance of mutation is dictated by special roulettes. After three generations, up to eight different DNA sequences could appear; (2) attribution of amino acids series to the eight nucleotide sequences in the third generation, following the genetic code; and (3) analysis of traits in these individuals and scoring of outcomes. To measure ACAGATATA's effect on learning, undergraduate students answered true-or-false questions before and after the game.
\end{abstract}

Results: In the tests, global scores after ACAGATATA were higher than before. Performance increased significantly in ten questions. Questions without direct connection to the game exhibited no significant change in performance. Satisfaction with the game was confirmed by high values in two questions asking the students about the contribution of ACAGATATA to their knowledge on the role of mutation in evolution.

Conclusions: ACAGATATA is suitable for biology courses concerning genetic information, its expression and molecular evolution, allowing an increase in student performance on these issues. Low cost justifies the adoption of ACAGATATA even by teachers with restricted resources.

Keywords: Biodiversity, Molecular evolution, Mutation, Phylogeny, Tree thinking

\section{Background}

The central claim of Darwin-Wallace's theory of evolution is the notion that all organisms descend with modification from common ancestors, since the first steps of life on Earth. Darwin and Wallace (1858) and Darwin (1859) suggested that the primary force of evolution is natural selection of preexistent varieties. This statement was severely debated. Morgan $(1925,1932)$ explained the roles of mutation and natural selection in shaping organismal evolution, based on Mendelian genetics (Nei 2005). In his view, advantageous mutations would persist while deleterious mutations would not. Following Morgan's work, molecular studies indicated the importance of random mutation in the evolutionary change of DNA.

\footnotetext{
* Correspondence: charlesmorphy@gmail.com Universidade Federal do ABC (UFABC), Centro de Ciências Naturais e Humanas, Rua Santa Adélia, 166, Bairro Bangu, Santo André, SP CEP 09210-170, Brazil
}

After the Modern Evolutionary Synthesis (1930s to 1940s), natural selection reassumed the main role in shaping biological diversity. The belief that every morphological or physiological character evolves by natural selection is still advocated by ultra-Darwinians (Eldredge 1995), who overemphasize its power on organismal features. Nonetheless, in the 1960s, Kimura (1968) proposed that the great majority of evolutionary changes at nucleotide level are caused by random genetic drift of selectively neutral mutants. Since every phenotypic trait is somehow genetically controlled, it is logical to think that a substantial part of changes in phenotypes must be caused by changes in DNA molecules. In short, there could be evolution without natural selection (by random drift), but there is no evolution without mutation (Nei 1975, 1987, 2005).

Hennig $(1950,1966)$ proposed a method of reconstructing phylogenies that revolutionized the view of the natural order of living beings, which is a product of evolutionary

\section{Springer}


processes. His phylogenetic systematics is based on the detection of exclusive features shared by two (sister) groups but absent in a third (out) group: the synapomorphies (Novick et al. 2010). Nowadays, nucleotide sequences are commonly used to hypothesize the evolutionary relationships among species or more inclusive groups. The history of mutations in DNA molecules as well as their consequences in phenotypic traits could be traced back by Hennigian phylogenetic trees.

Based on concepts of molecular biology and phylogenetic systematics, this paper presents the "Mutation Game" called ACAGATATA. The game simulates the evolution of related individuals along a few generations, in order to discuss how series of random mutations in DNA result in phenotypic variation. The rules of ACAGATATA combine fundamentals of DNA structure and genetic code with "tree thinking" (Baum et al. 2005; Meisel 2010). The aim of the game is to enable biology students to learn the process of descent with modification in a playful way.

\section{Methods}

How to play ACAGATATA

To play ACAGATATA, students should be aware of basic concepts of genetics and molecular evolution -for a review, see textbooks by Lehninger et al. (2008) and Lewin (2007). The role of randomness in the origin of biodiversity is the main theme of the game. Mutation is shown as an error in the copy of a single informative DNA strand, during the asexual reproduction of a haploid organism. Throughout consecutive generations of copies from its parental nucleotide sequence ACAGATATA, such errors are accumulated, leading to the production of different peptides, when compared to the one synthesized from the original DNA information. Hence, random changes in ancestral genotypes reflect on descendant phenotypes. The simulation is a didactic approach to concepts and mechanisms related to phenotypic expression, based on a simple model of molecular evolution.

Each player has his/her own board (printed on an A4 page) showing a phylogenetic tree to be constructed (Figure 1). It presents a segment of the "parental" DNA $\left(5^{\prime}-3^{\prime}\right.$, sense strand), that branches into two offspring, then four and, finally, eight "descendant" sequences of related individuals. The sequence ACAGATATA (generation "P") is the same for all players but the sequences that arise in the following three generations (F1 to F3) may be different, depending on the history of copy errors (i.e., mutations).

Players use two special roulettes to "induce" mutations (Figure 1, left). They are printed on the board, and a pencil could work as an axis for a paper clip kicked by a finger. The "Mutation Wheel" is divided into six sectors labeled as A (adenine), C (cytosine), G (guanine), T (timidine), + (plus sign), - (minus sign), and two sectors with an $\mathrm{N}$ mark (no mutation), all with the same size.
The "Position Wheel" is divided in equal sectors numbered from one to nine, signaling the exact position of the DNA sequence to be mutated.

The game has three phases:

\section{Phase 1: Replication}

The goal is to complete the diagram printed on the board (Figure 1) by submitting a sequence of DNA bases to successive copies. Errors (mutations) may occur at each reproduction event, at each generation (a round of copies). ACAGATATA corresponds to the ancestral DNA string, common for all players. During three generations of replication, possible mutations are determined by the outcome of roulette spins. Immediate ancestors (models for copies) and their immediate descendants (the two offspring, which are copies from the same model, but with different possibilities of errors) are connected by arrows. After three generations, up to eight different genotypes could appear.

The "Mutation Wheel" shows what will happen to the base indicated by the "Position Wheel" in a given ancestral DNA sequence submitted to replication. If the outcome of the first roulette is $\mathrm{N}$, the resulting descendant sequence remains as the previous one, i.e., a copy without mutation. It also happens when the outcome of the "Mutation Wheel" is the same base present in the sequence to be copied, exactly at the position pointed by the "Position Wheel". If the outcome is a minus sign (-), the base at the specified position should be removed and all the bases afterward should be relocated to early positions. The result is a copy with a deletion, and the resultant sequence is one-base shorter. If the outcome is a plus sign $(+)$, the "Mutation Wheel" should be spun until resulting in a base (A, C, G or T) to be included at the specified position, with all the bases afterward being one-position delayed. The resultant sequence is a copy with an insertion, which is one-base longer than its ancestral sequence. It is important to notice that a copy without mutation from an ancestral sequence (e.g., ACAGATATA) results in the same sequence (not in its complement, i.e., TGTCTATAT).

\section{Phase 2: Translation}

This phase occurs after completion of the tree in Figure 1. An amino acid should be ascribed to each base triplet of the DNA sequence (codon) at the last generation (F3). To simplify the game, the role of mRNA, tRNA and ribosomes in the translation of genetic information to amino acid series (peptides) is omitted in this simulation. An adapted, unconventional Genetic Code table (Figure 1, right top), directly relating the 64 possible triplets (codons) of the four DNA bases (A, C, G and T) to its 20 correspondent amino acids, is used during this phase. 


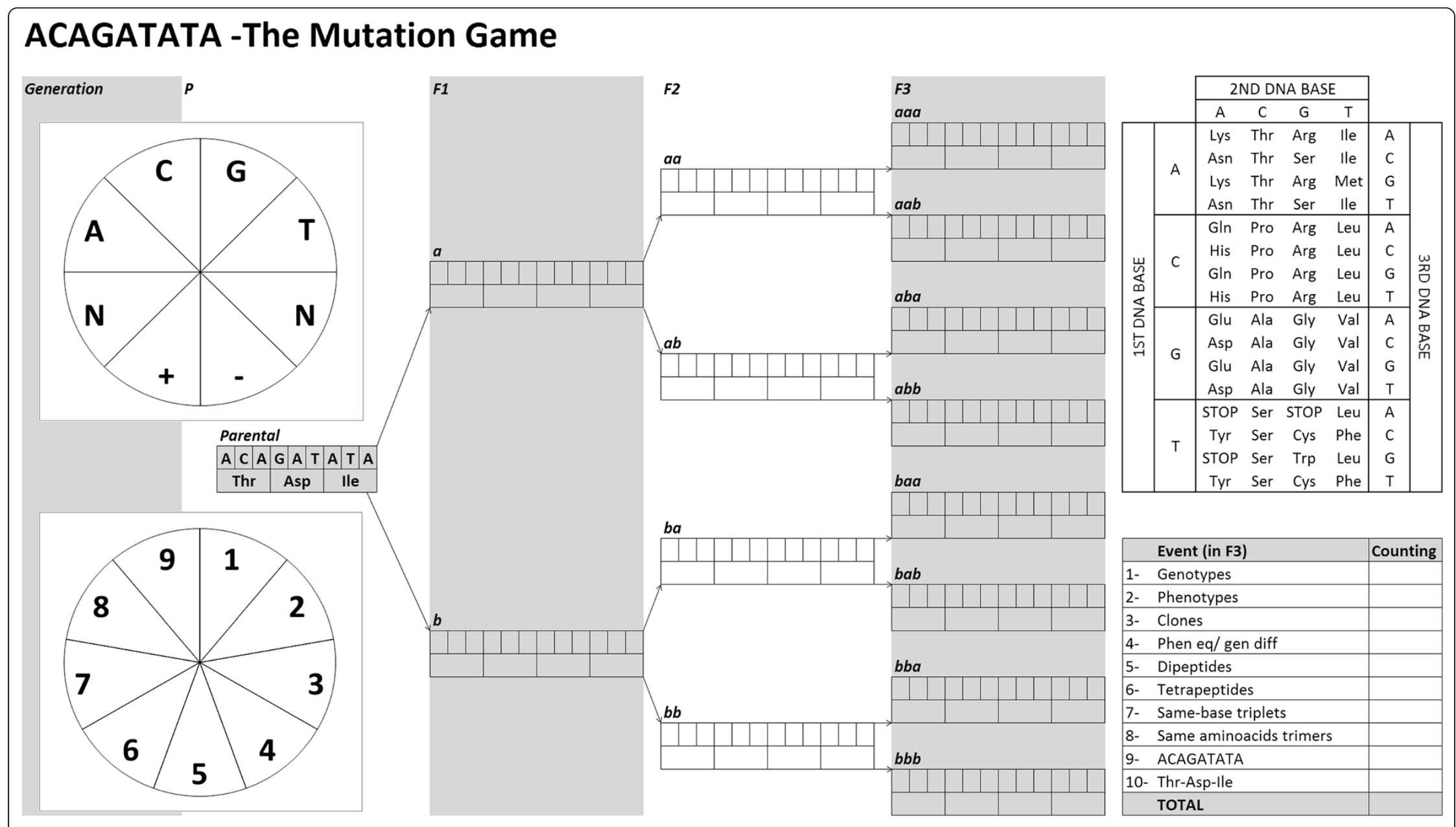

Figure 1 The game board. It shows a phylogenetic tree to be constructed, with the parental DNA sequence ACAGATATA (center left, generation "P") branching in two offsprings (in F1), then four (F2) and, finally, eight "descendant" sequences (F3). Also printed in the board are two special roulettes to "induce" copy errors ("Mutation Wheel", left top, and "Position Wheel", left bottom), an adapted, unconventional Genetic Code table (right top), and a score table (right bottom). 
Nonsense (TAA, TAG and TGA) or incomplete codons (at the end of strings, generated by insertions or deletions) are not translated. In F3, it is expected that lineages in which only substitutions occurred will originate peptides with three amino acids. Serial deletions can result in dipeptides; serial insertions can result in tetrapeptides. Combinations of substitutions, deletions and insertions along the generations can result in incomplete codons, with no correspondence to any amino acid. The pair formed by the base sequence and its respective amino acid sequence corresponds to the binomial genotypephenotype for each of the eight individuals at the last generation F3 (Figure 1).

\section{Phase 3: Interpretation}

The game ends with the comparison among the eight resultant individuals. A score is attributed according to ten events occurring during the simulation and detectable in the last generation F3 (see table at right bottom of Figure 1). These special occurrences are described as follows:

1. Different genotypes - the number of individuals with different base sequences. It effectively indicates how mutation could originate genetic diversity.

2. Different phenotypes - the number of individuals with different peptides. It shows how mutation could generate different amino acid sequences. To our didactical and reduced model, peptides are considered the ultimate phenotypes.

3. Clones - the number of identical genotypes present among the eight resultant sequences. It means that, even under different histories of sequential mutations, two or more individuals could exhibit the same genotype.

4. Equal phenotypes but distinct genotypes - the number of equal peptides presented by two or more individuals, but originated from distinct base sequences (i.e., originated by non-clones). It shows that changes in genetic information do not result in different phenotypes, given the redundancy of the genetic code.

5. Dipeptides - the number of individuals with only two-amino acid peptides as a phenotype. This event represents a process of evolutionary reduction in the size of the proteins, with repercussion on their spatial arrangement and, so, on their functional properties.

6. Tetrapeptides - the number of individuals with a four-amino acid peptide as a phenotype. It means the evolutionary increasing in the size of the protein.

7. Same-base triplets - the number of individuals with sequences composed only of some of the following codons: AAA, CCC, GGG or TTT. It simulates the origin of highly repeated DNA sequences, whose function is debated.
8. Same amino acids trimers - the number of individuals with a tripeptide composed of only a single kind of amino acid.

9. ACAGATATA - the number of individuals who maintain the original sequence of the game. As well as the absence of mutations, errors followed by reversals could result in a descendant genetically identical to their ancestor.

10.Thr-Asn-Ile - the number of individuals with the tripeptide Threonine-Asparagine-Isoleucine (ACAGATATA's phenotype). Errors followed by reversals or absence of mutations could result in a descendant with the same phenotype as their ancestor. However, as seen before, even genetically different individuals could produce the same peptide; the genetic code is redundant.

After examining the eight resultant sequences in search for the events summarized above and the sum of all of them, the player with the highest score in the classroom is the winner. However, the main aim is not simply to achieve the highest score, especially because the result will be fundamentally dependent on chance and not on special skills of the players, but to exercise students' knowledge on important aspects about the role of random processes in molecular evolution. This exercise can be carried out individually or in small groups (up to three persons per board), depending on the number of students in the classroom. In such groups, each round can be discussed among the components, minimizing possible errors when filling out the board and during the interpretation of the resulting sequences. The planned time for the game is one hour for explanations on essential topics (e.g., the nature of randomness in molecular evolution and the basis of phylogenetic reasoning) and an additional hour for the game itself, including discussion of relevant outcomes. As discussed above, previous knowledge of replication, transcription and translation processes are welcome, but even when this knowledge is lacking, the game itself is useful to introduce such key concepts in the classroom. Student performance can be evaluated by standard written examination.

\section{Results}

\section{Studying the expected outcomes}

To predict what teachers in the classroom will find in the student's board game, we performed a study of outcome probabilities for each event that scores in ACAGATATA. In an Excel worksheet, we simulated 10,000 board fillings, according to (i) the rules dictated by the "Mutation" and the "Position Wheels" during the filling out of the diagram, and (ii) the translation of the eight terminal DNA sequences (genotypes) to amino acid series (phenotypes) according to the genetic code. The probability distributions 
(a)

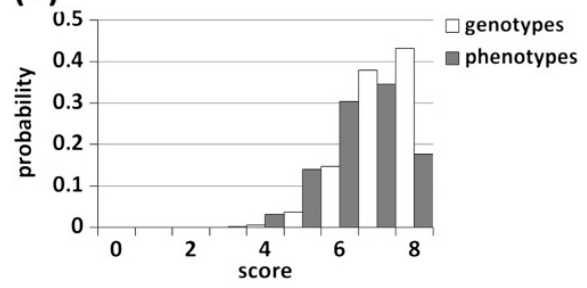

(c)

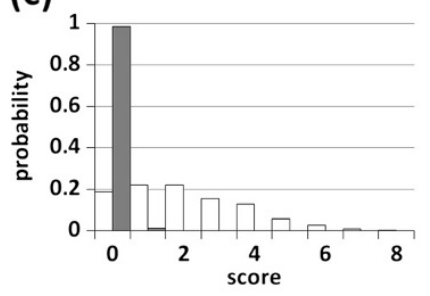

(e)

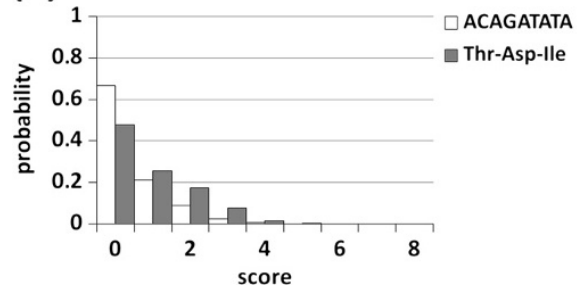

(b)

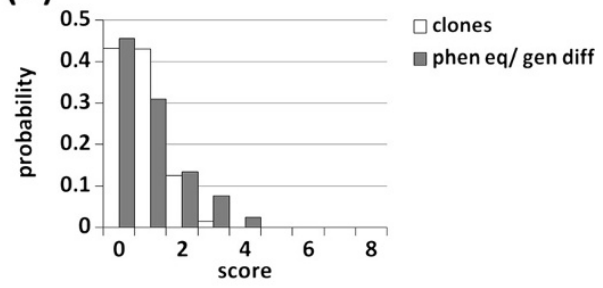

(d)
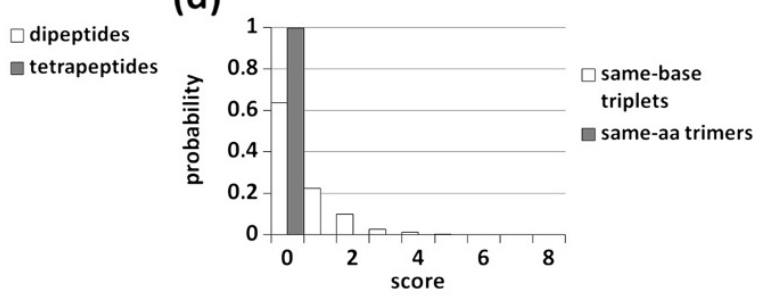

(f)

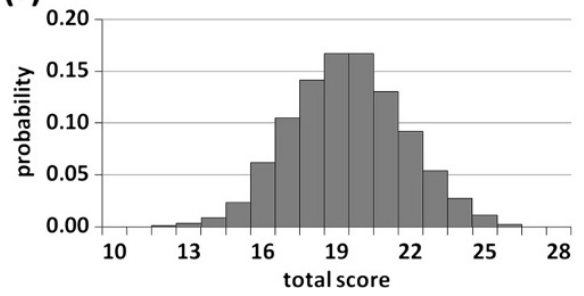

Figure 2 Probability distribution of outcomes for ten events, based on Excel worksheet simulations. (a) [genotypes] = number of individuals with different base sequences and [phenotypes] = number of individuals with different amino acid sequences (different peptides); (b) [clones] = number of genotypes present in more than one individual and [phen eq/gen diff] = number of peptides presented by more than a single individual, but originated from distinct sequences; (c) [dipeptides] = number of individuals with a two-amino acid peptide as phenotype and [tetrapeptides] = number of individuals with a four-amino acid peptide as phenotype; (d) [same-base triplets] = number of individuals with codons AAA, CCC, GGG, or TTा and [same-aa trimers] = number of individuals with a tripeptide formed by only one kind of amino acid; (e) $[$ ACAGATATA] = number of individuals with the original sequence and [Thr-Asp-lle] = number of individuals with the tripeptide ThreonineAsparagine-Isoleucine (ACAGATATA's phenotype); ( $\mathbf{f}$ ) total score (sum of all scores at each board).

of the results for the events take in account in the score table and global results are presented in Figure 2.

Analyzing the eight individuals in each "virtual board" at the end of a match, $43 \%$ of the simulations resulted in eight different genotypes (Figure 2a). In 38\%, seven different genotypes appeared among those individuals (with one repetition of a DNA sequence). More than one genotype repetition occurred in less than $19 \%$. The number of phenotypes of such individuals has a distinct distribution: $18 \%$ of the simulations resulted in eight different amino acids series, 35\% presented seven phenotypes (repetition of one peptide out of eight), and more than $47 \%$ of the matches have two or more repetitions of phenotypes (Figure 2a). As expected, the phenotype (represented by an amino acid series or peptide) is more conservative to changes (mutation) than the genotype. It is easily explained. The genetic code is said to be "degenerated", i.e., an amino acid can be included in a peptide by information from different codons.
Among the eight individuals in the last generation, clones were absent in $43 \%$ of the simulations (Figure $2 \mathrm{~b}$ ). Clones of a single DNA sequence were presented in the same proportion. Clones of more than one genotype appeared in less than $14 \%$ of the results. Individuals with the same phenotype but different genotypes were presented in $54 \%$ of the matches (Figure $2 b$ ).

Dipeptides originated from sequential deletions through the generations were frequent (81\%), but tetrapeptides arising from sequential additions were rare (1.4\%) (Figure 2c). Triplets of a given base (AAA, CCC, GGG, TTT) appeared in $36 \%$ of the simulations (Figure $2 \mathrm{~d}$ ). Triplets of the same amino acid were extremely infrequent (less than $0.2 \%$ ) (Figure 2d).

Even after three generations from the "parental" sequence, the genotype ACAGATATA appeared at least once in $33 \%$ of the simulations, but its phenotype Thr-Asp-Ile was more common (52\%) (Figure 2e). Finally, the highest and the lowest global score in the game were, respectively, 
Table 1 Test applied to 109 undergraduate students at UFABC, Brazil

\begin{tabular}{|c|c|c|c|c|c|c|}
\hline Number & Question & $\mathrm{P}_{\mathrm{b}} \%$ & $P_{a} \%$ & $x^{2}$ & $P$ & \\
\hline 1 & Mutation occurs during DNA replication. [T] & 71.6 & 91.7 & 21.8 & 0.001 & ** \\
\hline 2 & Mutation occurs during DNA transcription. [F] & 33.0 & 45.0 & 7.0 & 0.008 & * \\
\hline 3 & Mutation occurs during messenger-RNA translation. [F] & 47.7 & 60.6 & 7.2 & 0.007 & * \\
\hline 4 & Mutation occurs during protein synthesis. [F] & 73.4 & 71.6 & 0.2 & 0.665 & \\
\hline 5 & Mutation is a DNA polymerase error. [T] & 56.0 & 48.6 & 2.4 & 0.123 & \\
\hline 6 & Mutation is a RNA polymerase error. [F] & 37.6 & 54.1 & 12.7 & 0.001 & ** \\
\hline 7 & Mutation is a ribosome error. [F] & 86.2 & 88.1 & 0.3 & 0.578 & \\
\hline 8 & Mutation is a transfer-RNA error. [F] & 59.6 & 72.5 & 7.5 & 0.006 & * \\
\hline 9 & The point in the molecule where a mutation will happen is unpredictable. [T] & 90.8 & 97.2 & 5.4 & 0.020 & * \\
\hline 10 & Environmental conditions increase the chance of mutation. [T] & 59.6 & 56.9 & 0.3 & 0.558 & \\
\hline 11 & Mutation always results in a new genotype. [T] & 74.3 & 74.3 & 0.0 & 1.000 & \\
\hline 12 & Mutation always results in a new phenotype. [F] & 79.8 & 93.6 & 12.8 & 0.001 & ** \\
\hline 13 & Individuals with the same genotype are clones. [T] & 69.7 & 97.2 & 39.1 & 0.001 & ** \\
\hline 14 & Individuals with the same phenotype are clones. [F] & 81.7 & 90.8 & 6.1 & 0.013 & * \\
\hline 15 & Mutation generates individuals with low fitness. [T] & 14.7 & 19.3 & 1.8 & 0.176 & \\
\hline 16 & Mutation generates individuals with high fitness. [T] & 22.9 & 21.1 & 0.2 & 0.649 & \\
\hline 17 & Mutation generates genotypic diversity. [T] & 94.5 & 97.2 & 1.6 & 0.208 & \\
\hline 18 & Mutation generates phenotypic diversity. [T] & 70.6 & 79.8 & 4.4 & 0.035 & ${ }^{*}$ \\
\hline 19 & ACAGATATA contributed for the knowledge on genetic expression. & & 97.3 & & & \\
\hline 20 & ACAGATATA contributed for the knowledge on evolution. & & 87.2 & & & \\
\hline
\end{tabular}

Group performance in each of 18 true-or-false questions (correct T or $\mathrm{F}$ answer in brackets) was compared by chi-square tests in $2 \times 2$ contingency tables with the number of students that hit or missed each answer, before and after the game. One asterisk denotes significant $(a=0.05)$ chi-square values $\left(X^{2}, d f=1\right)$. Two asterisks denote values significant after a Bonferroni correction for the number of tests $\left(\alpha_{c}=0.05 / 18=0.003\right) . P_{b} \%$ and $P_{a} \%$ are percent hits of the group before and after the game, respectively. An a priori overall test comparing individual scores in the entire questionnaire indicates a significant increasing in global performance after ACAGATATA (medians: $\mathrm{P}_{\mathrm{b}} \%=66.7 \%$ and $\mathrm{P}_{\mathrm{a}} \%=72.2 \%$; Wilcoxon $\mathrm{W}=2754, P=0.004, \mathrm{~N}=109$ ). The last two questions were applied only at the second turn.

28 and 10 points (median $=19)$ (Figure $2 \mathrm{f}$ ), so allowing a distribution of the students in 19 ordered categories based on their outcomes.

\section{ACAGATATA's effect on learning}

Undergraduate students $(\mathrm{N}=109)$ played ACAGATATA at the start of a discipline on evolution, during a Science and Technology course at the Universidade Federal do $\mathrm{ABC}$, Brazil. Just before explaining the rules, we gave the group a test with 18 true-or-false questions concerning general topics on genetic information and its expression (Table 1). These questions are correlated in different degrees to the theoretical basis required by ACAGATATA. Immediately after the game (which lasts 1.5 hours, including exposition of rules and all of its three phases) we re-administered the same test. Global scores after ACAGATATA (median of individual hits $=72.2 \%$ ) were significantly higher than before (median of individual hits $=66.7 \%$ ) (Wilcoxon $\mathrm{W}=2754, P=0.004, \mathrm{~N}=109$ ). A posteriori, we analyzed the scores for each question individually in a $2 \times 2$ contingency table (number of students with right $\mathrm{x}$ wrong answers, before $\mathrm{x}$ after the game).
Results before the game were used as expected values in chi-square tests, the null hypotheses being "no effect of ACAGATATA in effective learning about mutation and molecular evolution". Performance increased significantly in ten questions (Table 1). In four questions $(1,6,12$ and 13) the results remained significant after a Bonferroni correction for the number of tests $(P<0.05 /$ $18=0.003)$. In two additional questions at the second evaluation, students reported the contribution of ACAGATATA for their ideas on genetic expression (97.2\%) and evolution (87.2\%).

These analyses indicate that the game ACAGATATA improved students' knowledge of:

(i). The circumstances in which a mutation occurs during DNA replication and, consequently, the role of some entities involved in this copy process and in the expression of genetic information (questions 1, 2, 3, 6, 8 and 9);

(ii). the mutation effect on phenotypic expression and biodiversity generation (questions 12 and 18); and

(iii).the ideal definition of clones (questions 13 and 14). 
Questions without direct connection to the ACAGATATA theoretical basis act as a kind of "experimental control", exhibiting no significant change in performance, as expected. The effect of mutation on fitness (questions 15 and 16), and the conditions which increase the chance of error (question 10) are issues not addressed by the game.

Satisfaction with both the game and its purpose was verified by high values in two questions asking the students about the contribution of ACAGATATA to their knowledge of genetic expression and evolution (Table 1).

\section{Discussion}

\section{Some important remarks}

The board game ACAGATATA is entirely focused on the effects of random mutations in generating genetic and phenotypic diversity. However, as a model, it is simpler than the real evolutionary process. In fact, it was based on a reductionist view of evolution. For instance, it does not consider natural selection. The game presents each student with the same nine-nucleotide sequence, which they follow through three generations. During each round of copies, random mutations are introduced by the spin of special roulette wheels. The eight sequences produced after these three rounds of replication are then scored in search of a variety of special events.

As a game designed to simulate the role of mutations in molecular evolution, ACAGATATA makes a number of assertions. First, the simulation is valid for an asexual, haploid organism, in which there are no genetic novelties generated by the recombination of parental genotypes during meiosis, and there are no interactions of pairs of alleles to express a given trait. Each individual has only two offspring, with independent histories of DNA copy from such an ancestor.

Second, the roulette wheels create a very high chance that a mutation occurs in each generation $(5 / 8=62.5 \%)$, which is higher than mutation rates occurring in nature. As stated by Lynch et al. (1999), the average mutation rate per base pair has been estimated at around $10^{-11}$ or $10^{-10}$ per replication in prokaryotes and $10^{-9}$ per sexual generation in eukaryotes. According to Futuyma (2005, p. 172), "with such a low mutation rate per locus, it might seem that mutations occur so rarely that they cannot be important. However, summed over all genes, the input of variation by mutation is considerable". Extrapolating the mutation rates to high values is a form of guarantee of the occurrence of mutations during the rounds of the game and, hence, to introduce to students the importance of changes in genetic material as one of the causes of the great variability (both genetic and phenotypic) found in the natural environment.

Third, our approach does not consider the transcription of DNA information to the mRNA molecule, or the role of tRNA and ribosomes in translation. The adapted Genetic
Code table makes a direct link between the DNA codons in a sense strand and the primary structure of the resultant peptide. Students should be aware that the conventional Genetic Code relates mRNA codons to amino acids and that the template for such mRNA during transcription is the anti-sense DNA strand. Our aim is to present only the basic tenets of evolution at the molecular level, and such type of simplification - a close relationship between the DNA information and the peptide structure - is necessary in the context of this board game.

Fourth, ACAGATATA also demands the interpretation of each resultant amino acid sequence as a phenotype. The peptide (also called "protein" in the game) is the ultimate expression of the genotype. This is obviously an approximation because changes in the primary structure of a protein often lead to non-detectable (phenotypic) variations of morphological or physiological attributes. Actually, given the regulatory system of gene expression also omitted in this simulation, it is possible for a specific genotype to generate different phenotypes, (e.g., a nerve cell and a muscle cell from the same individual share the same genotype but they show quite different features). For the purposes of the game, however, we decided to draw a very tight correspondence between genotype and phenotype.

Finally, to maintain the characteristics of a board game, it would be necessary to provide some kind of score to determine the winner. Therefore, we chose some events to compose our score values, which were determined based on the probability of obtaining each DNA sequence and its resulting amino acid sequence, as shown in Figure 2. Outcomes related to evolutionary changes in the size of these molecules or to the origin of repeated monomers were contemplated. We primarily focused the relationship between genotypes and phenotypes. In this context, we defined clones as genetically and strictly identical individuals.

\section{Avoiding misconceptions}

This section discusses some of ACAGATATA's limitations as a model for teaching molecular evolution, precluding pitfalls due to misconceptions on which skills the game intends to exercise. Already discussed in other parts of the text, the following warnings are particularly necessary when students have no previous knowledge of processes involving informational molecules. They are equally welcome even for those students that have prior contact with such biochemical issues, to avoid confusion between mechanisms in the real world and the form on which the game was built. ACAGATATA is a simple model of complex processes that happen in nature. It over-reduces or even omits some factors, inflating the contribution of other (selected) target factors. Examples of similar representation of evolutionary processes, reductionist models of a complex reality, are the classic biomorphs 
(Dawkins 1986) and the legorgs (Christensen-Dalsgaard and Kanneworff 2009).

i. ACAGATATA does not deal with natural selection. In ACAGATATA, biological evolution is shown as a plausible result of random effects solely, without selective forces. All individuals (genotype + phenotype) have the same chance to survive and reproduce as their parents, contributing equally to the gene pool in further generations as dictated by the roulettes. Hence, ACAGATATA suppresses natural selection, highlighting the role of randomness in biodiversity generation. In the real world, interaction among genotypes and their environment will eliminate some forms, allowing a high chance of reproduction for the other remaining forms. So, in ACAGATATA, randomness is obviously shown as an entity detached from selection, i.e., mutation is undirected by selective pressures, as occurs in nature. Selective effects on diverse forms could be taught using alternative models as the aforementioned biomorphs or legorgs.

ii. Mutation rate is highly inflated in ACAGATATA. In ACAGATATA, mutation probability is the same along a DNA segment submitted to sequential copying rounds. Substitutions are equally probable among bases, as well as insertions and deletions. This simplification has operational purposes, given the natural occurrence of DNA sites prone to copy errors, besides commoner types of molecular changes. When compared to natural values, the mutation rate in ACAGATATA is inflated, as a strategy to allow students to see its effects in their own (small) set of individuals through (few) generations. Generally, mutation is only detected in natural populations with a high number of individuals, after many generations.

iii. ACAGATATA's "Replication phase" does not directly correspond to DNA replication process.

The "Replication phase" in ACAGATATA should not be treated as a facsimile of the DNA replication process. The real process is "embedded" in each copying round of a DNA template that results in one of its descendant sequences in the game. The other descendant from the same parent is generated by another independent copying round (that also implies an event of DNA replication). Each of these two copying rounds is independently susceptible to errors (mutations). As original DNA information could be corrupted at each round of copy and once an error occurs, the new information has its own turn of reproduction, under a given chance of a new, independent error. Occasionally, the original information could arise again after a series of counteracting mutations, e.g., an insertion following a deletion at the same point. Repairing processes are also omitted in ACAGATATA. The game focuses a coding region of a protein-encoding gene. However, in nature there are regulatory regions, splicing sites, and other non-coding DNA sectors also "available" for copying errors, with possible effects on resulting phenotypes through generations. The game does not account for these questions and their evolutionary consequences.

iv. ACAGATATA's "Transcription phase" does not directly correspond to mRNA transcription process. ACAGATATA adopts a special table to make a direct conversion of DNA information to an amino acid sequence. All books present the gene code table relating mRNA codons to a given amino acid. This translation process (mRNA to protein, via interaction of ribosomes and tRNA) is omitted in the game, as well as the previous transcription process (DNA to mRNA); both are embedded in ACAGATATA's "Translation phase". Our "unconventional" table is a simple resource to relate genetic information to what we call the individual phenotype, a corresponding peptide. There is no theoretical compromise in shortening two consecutive steps of information conversion. This process preserves information integrally, as could be seen during reverse transcription (mRNA to DNA) adopted in cDNA techniques. One could find identical "DNA to protein conversion" tools on the internet. The way users acquire a correspondent amino acid for a given DNA codon in ACAGATATA table is exactly the same as with a conventional table, i.e., by crossing specific columns and rows to find an output cell with the desired information.

v. ACAGATATA uses a peptide as the ultimate phenotypic manifestation of each genotype. Based on the statement "one gene - one protein", ACAGATATA relates each series of DNA bases (individual genotype) to a series of amino acids (individual phenotype). Here, genic expression is not subjected to any regulatory mechanisms. Interactions between such output and environmental conditions are omitted, and all individuals are equally viable to survive and to reproduce. In the real world, genetic information is expressed in different ways, depending on internal control or external conditions, sometimes leading to very distinct phenotypes. Different phenotypes generally present different performances in a given set of environmental conditions, which contribute to differential fitness among individuals. In nature, the fittest individuals are those that have a majoritarian contribution to the gene pool in the next generation. 


\section{Overview}

It is widely known that the theory of evolution is the current unifying paradigm of biological sciences and the research program with the greatest explanatory power throughout biological disciplines (Futuyma 1999; Mayr 2000; Gould 2002 and so on). In fact, the revolution initiated by Darwin and Wallace held considerable sway beyond biological disciplines. Evolutionary reasoning has actually influenced human thinking from biology to the philosophy of science, providing a materialistic view to explain the living world. It is hard to find an aspect of modern mankind not affected by such principles (Mayr 2000). As the theory of evolution is the fundamental pillar of modern biology, it seems obvious to use it as the organizing theme in teaching biology beginning in the early elementary grades (Nadelson et al. 2009). Besides, evolution brings to the school a broader perspective on natural phenomena and on the nature of scientific activity (Tidon and Lewontin, 2004; Santos and Calor, 2008; O’Brien et al. 2009).

Unfortunately, as pointed out by Pazza et al. (2010) and many others, there remains a widespread lack of understanding about evolutionary theory, which is still quite susceptible to misunderstandings. In Brazilian schools, for example, the theory is often presented to students in an oversimplified manner in just a few class sessions, usually during the last year of their biology courses. Notwithstanding, the emphasis is placed especially on the struggle between Lamarck's and Darwin's views on the evolutionary process. Sometimes, natural selection is considered. The role of stochastic events in generating biodiversity, however, is seldom treated properly. In order to deal with evolutionary theory in a wide perspective, it is essential to discuss evolution beyond Lamarck, Darwin or Wallace, and even beyond natural selection. Discussing the importance of random mutations and the basis of molecular evolution in biology classes complements students' views of the evolutionary process. Phylogenetic hypotheses are powerful tools in this context. Herein, phylogenetic reasoning was used to represent the comparison among different DNA sequences derived from an ancestral DNA sequence, ACAGATATA.

A board game involves the active participation of students in the construction of knowledge, which is different from simple reconstruction of previously elaborated knowledge provided by the teacher or by the textbook (Gil-Pérez et al. 2002). The "Mutation Game" proposed here is a simple (but not superficial) manner to present how molecular evolution proceeded to shape the "tree of life". Using a reductionist model that mimics the role of random mutational processes in the emergence of biodiversity, this board game is a device for initiating and training students in the study of DNA and its relation to evolution. In spite of its widespread application, molecular biology is a research field hardly known by the majority of life sciences students, both in its theoretical bases and in its technical approaches (Cardona et al. 2007), thus requiring new teaching strategies (Cardoso et al. 2008).

\section{Conclusion}

Despite some simplifications (a nine-base-long DNA strand to represent a genotype, or the tight link between genotype and phenotype) and some omissions (e.g., the role of different RNAs in transcription and translation, the role of natural selection), the simulation shows how the random nature of mutation is essential for the generation of diversity at different organismal levels. Mutation appears as a stochastic event that may change the expected outcome - a copy without modification - given the efficiency and correctness of the replication system through the action of DNA polymerases. Compared to the real world, the mutation rate considered in the game is very high, as arbitrarily set by the previous design of the roulettes. For instance, the probability that the original sequence ACAGATATA remains unchanged after three generations in a single lineage (e.g., a, ab, abb) is very small (6.19\%). It implies that the student would frequently witness a mutation and its effects on the generation of diversity in his/her own board. In this sense, as pointed out before, it is possible to introduce the evolutionary importance of randomness in the generation of biological diversity, a perspective far away from the adaptationist reasoning commonly used to explain why there are so many different species in the world. The aim of the game ACAGATATA is not to deny natural selection as one of the major forces of evolution. Indeed, the game points to the need for more complex discussions about the evolutionary process emphasizing an often neglected topic - the role of mutation - in secondary and undergraduate biology courses.

A remarkable feature of the game is its low cost (one printed A4 page per student), allowing its use even in conditions of restricted resources. When previous ACAGATATA versions were given to our students in evolution classes, it was received by them as a fun way to learn a considerably hard (and, sometimes, boring) topic in biology. Statistical evaluation of student performance after the game and their perceptions during the activity reinforces our statements.

\section{Competing interests}

The authors declare that they have no competing interests.

\section{Authors' contributions}

$\mathrm{AE}$ conceived the game and performed the statistical analysis. CMDS led text writing. Both authors designed the study and gathered data. Both authors read and approved the final manuscript.

\section{Acknowledgements}

The authors would like to thank Gisele Cristina Ducati (CMCC-UFABC) and two anonymous reviewers for criticisms and suggestions on an early version 
of this manuscript. Comments from Vivian Leyser da Rosa during the III ENEBIO (Fortaleza, Brazil/2010) help to improve our text. The name

ACAGATATA is homage to Otavio "Tatá" Marques (Instituto Butantan, Brazil).

Received: 11 March 2013 Accepted: 11 March 2013

Published: 13 May 2013

\section{References}

Baum, DA, Smith, SD, \& Donovan, SSS (2005). The tree-thinking challenge. Science, 310(5750), 79-80.

Cardona, TS, Spiegel, CN, Alves, GG, Ducommun, J, Henriques-Pons, A, \& AraujoJorge, TC (2007). Introducing DNA concepts to Swiss high school students based on a Brazilian educational game. Biochemistry and Molecular Biology Education, 35(6), 416-421.

Cardoso, FS, Dumpel, R, da Silva, LB, Rodriques, CR, Santos, DO, Cabral, LM, et al. (2008). Just working with the cellular machine: a high school game for teaching molecular biology. Biochemistry and Molecular Biology Education, $36(2), 120-124$

Christensen-Dalsgaard, J, \& Kanneworff, M (2009). Evolution in Lego ${ }^{\circledR}$ a physical simulation of adaptation by natural selection. Evolution: Education and Outreach, 2(3), 518-526.

Darwin, C, \& Wallace, A (1858). On the tendency of species to form varieties, and on the perpetuation of varieties and species by natural means of selection. Journal of the Proceedings of the Linnean Society. Zoology, 3(9), 45-62.

Darwin, C (1859). On the origin of species by means of natural selection or the preservation of favored races in the struggle for life. London: John Murray.

Dawkins, R (1986). The blind watchmaker. New York, NY: Norton \& Company.

Eldredge, N (1995). Reinventing Darwin: the great debate at the high table of evolutionary theory. New York: John Wiley \& Sons.

Futuyma, DJ (1999). Evolution, science and society: evolutionary biology and the national research agenda. New Brunswick, NJ: The State University of New Jersey.

Futuyma, DJ (2005). Evolution. Sunderland, MA: Sinauer Associates, Inc., Publishers.

Gil-Pérez, D, Guisasola, J, Moreno, A, Cachapuz, A, de Carvalho, AM, Torregrosa, $J M$, et al. (2002). Defending constructivism in science education. Science \& Education, 11(6), 557-571.

Gould, SJ (2002). The structure of evolutionary theory. Cambridge, MA: The Belknap Press of Harvard University Press.

Hennig, W (1950). Grundzuge einer Theorie der phylogenetischen Systematik. Berlin: Deutscher Zentralverlag.

Hennig, W (1966). Phylogenetic systematics. Urbana: University of Illinois Press. Kimura, M (1968). Evolutionary rate at the molecular level. Nature, 217(5129) 624-626.

Lehninger, A, Nelson, DL, \& Cox, MM (2008). Lehninger principles of biochemistry (5th ed.). New York: WH Freeman.

Lewin, B (2007). Genes IX. Sudbury, MA: Jones and Bartlett Publishers.

Lynch, M, Blanchard, J, Houle, D, Kibota, T, Schultz, S, Vassilieva, L, et al. (1999). Perspective: spontaneous deleterious mutation. Evolution, 53(3), 645-663.

Mayr, E (2000). Darwin's influence on modern thought. Scientific American $283(1), 66-71$

Meisel, RP (2010). Teaching tree-thinking to undergraduate biology students. Evolution: Education and Outreach, 3(4), 621-628.

Morgan, TH (1925). Evolution and genetics. Princeton, NJ: Princeton University Press.

Morgan, TH (1932). The scientific basis of evolution. New York, NY: WW Norton

Nadelson, L, Culp, R, Bunn, S, Burkhart, R, Shetlar, R, Nixon, K, et al. (2009). Teaching evolution concepts to early elementary school students. Evolution, Education and Outreach, 2(3), 458-473.

Nei, M (1975). Molecular population genetics and evolution. Amsterdam: North-Holland Publishing Company.

Nei, M (1987). Molecular evolutionary genetics. New York, NY: Columbia University Press.

Nei, M (2005). Selectionism and neutralism in molecular evolution. Molecular Biology and Evolution, 22(12), 2318-2342. Erratum in: Molecular Biology and Evolution (2006) 23(5):1095.

Novick, LR, Catley, KM, \& Funk, DJ (2010). Characters are key: the effect of synapomorphies on cladogram comprehension. Evolution: Education and Outreach, 3(4), 539-547.

O'Brien, DT, Wilson, DS, \& Hawley, PH (2009). "Evolution for everyone": a course that expands evolutionary theory beyond the biological sciences. Evolution: Education and Outreach, 2(3), 445-457.
Pazza, R, Penteado, PR, \& Kavalco, KF (2010). Misconceptions about evolution in Brazilian freshmen students. Evolution: Education and Outreach, 3(1), 107-113.

Santos, CMD, \& Calor, AR (2008). Using the logical basis of phylogenetics as the framework for teaching biology. Papéis Avulsos de Zoologia, 48(18), 199-211.

Tidon, R, \& Lewontin, RC (2004). Teaching evolutionary biology. Genetics and Molecular Biology, 27(1), 124-131.

doi:10.1186/1936-6434-6-22

Cite this article as: Eterovic and Santos: Teaching the role of mutation in evolution by means of a board game. Evolution: Education and Outreach 2013 6:22.

\section{Submit your manuscript to a SpringerOpen ${ }^{\odot}$ journal and benefit from:}

- Convenient online submission

Rigorous peer review

- Immediate publication on acceptance

- Open access: articles freely available online

- High visibility within the field

- Retaining the copyright to your article

Submit your next manuscript at $\gg$ springeropen.com 